\title{
Geometric Stopping of a Random Walk and Its Applications to Valuing Equity-linked Death Benefits
}

\author{
Hans U. Gerber, Elias S. W. Shiuł Hailiang Yang ${ }^{\ddagger}$
}

\begin{abstract}
We study discrete-time models in which death benefits can depend on a stock price index, the logarithm of which is modeled as a random walk. Examples of such benefit payments include put and call options, barrier options, and lookback options. Because the distribution of the curtate-future-lifetime can be approximated by a linear combination of geometric distributions, it suffices to consider curtate-futurelifetimes with a geometric distribution. In binomial and trinomial tree models, closed-form expressions for the expectations of the discounted benefit payment are obtained for a series of options. They are based on results concerning geometric stopping of a random walk, in particular also on a version of the Wiener-Hopf factorization.
\end{abstract}

Keywords: Equity-linked death benefits, binomial and trinomial tree models, random walk, geometric stopping, Esscher transform.

\author{
JEL Classification: $\quad$ G13 G22 C02 \\ Subject Categories: $\quad$ IM10 IE50 IM40 IB10
}

\section{Introduction}

This paper is dedicated to the celebration of the 65th birthday of Professor Rob Kaas. A key motivation for this paper is the problem of valuing Guaranteed Minimum Death Benefits (GMDB) in various equity-indexed and variable annuity contracts. In the literature, the problem is usually studied in a continuous-time setting, e.g., Milevsky and Posner (2001), Ulm (2006, 2008), and Gerber et al. (2012, 2013). In practice, many actuaries use discrete-time models (International Actuarial Association 2010). One reason for this

\footnotetext{
${ }^{*}$ Department of Statistics and Actuarial Science, The University of Hong Kong, and Faculty of Business and Economics, University of Lausanne, CH-1015 Lausanne, Switzerland, e-mail: hgerber@unil.ch.

${ }^{\dagger}$ Department of Statistics and Actuarial Science, The University of Iowa, Iowa City, Iowa 52242-1409, U.S.A., e-mail: elias-shiu@uiowa.edu

${ }_{\ddagger}$ Corresponding author, Department of Statistics and Actuarial Science, The University of Hong Kong, Pokfulam Road, Hong Kong, e-mail: hlyang@hku.hk.
} 
is that insurance products are monitored periodically. We consider discrete-time models in this paper.

For $t=0,1,2, \ldots$, we model the time- $t$ price of a stock or stock index as

$$
S(t)=S(0) a^{X(t)}
$$

where $a>1$ is a constant and $\{X(t)\}$ is a random walk. If $\{X(t)\}$ is a simple random walk, then (1.1) is the binomial tree model popularized by Cox et al. (1979). In this paper, many explicit results are derived for binomial and trinomial tree models. For ease of discussion, the length of each period is usually assumed to be one year.

We are interested in evaluating expectations of the form

$$
\mathrm{E}\left[v^{K_{x}+1} b\left(S(0), S(1), \ldots, S\left(K_{x}\right)\right)\right],
$$

where $K_{x}$ is the curtate-future-lifetime (time until the beginning of the period of death) random variable for a life-age- $x$, and $b$ is a death benefit function that may depend on the stock-price history up to time $K_{x}$. We assume that the death benefit is paid at the end of the period of death.

Let us give some examples. Consider a GMDB rider that guarantees the following death benefit payment,

$$
\max \left(S\left(K_{x}\right), G\right)
$$

where $G$ is the guaranteed amount. Because

$$
\max \left(S\left(K_{x}\right), G\right)=S\left(K_{x}\right)+\left[G-S\left(K_{x}\right)\right]_{+},
$$

the problem of valuing the guarantee becomes the problem of valuing

$$
\left[G-S\left(K_{x}\right)\right]_{+},
$$

the payoff of a put option. Since $K_{x}$ is a random variable, the put option is of neither the European style nor the American style. It is a life-contingent put option (whose valuation in a binomial or trinomial tree model can be obtained by applying formula (7.10) below). Next, suppose that $G$ is not a constant but a fraction, say $90 \%$, of the maximum stock price from time 0 to time $K_{x}$. Then the problem is to value the payoff

$$
\left[0.9 \times \max \left(S(0), S(1), \ldots, S\left(K_{x}\right)\right)-S\left(K_{x}\right)\right]_{+} .
$$

This path-dependent option is called a fractional floating strike lookback put option (which can readily be evaluated by applying formula (9.19) below). Our third example arises from the fact that if the stock price rises, a put option such as (1.4) becomes less valuable and the policy may lapse. Thus, instead of (1.4) we may want to consider the payoff

$$
I_{\left(\max \left(S(0), S(1), \ldots, S\left(K_{x}\right)\right)<H\right)} \times\left[G-S\left(K_{x}\right)\right]_{+},
$$

where $I_{(.)}$denotes the indicator function and $H$ is a barrier. This is the payoff of an up-and-out put option, a particular form of barrier options that will be studied in Section 8. 
To evaluate (1.2), we assume that the random variable $K_{x}$ and the stock price process $\{S(t)\}$ are independent. As shown in Section 5, the distribution function of $K_{x}$ can be approximated by linear combinations of geometric distributions. Hence, our problem can be reduced to the evaluation of

$$
\mathrm{E}\left[v^{\tau+1} b(S(0), S(1), \ldots, S(\tau))\right]
$$

where $\tau$ is an arbitrary geometric random variable independent of $\{S(t)\}$. Also, (1.7) can be factorized as

$$
\mathrm{E}\left[v^{\tau+1}\right] \tilde{\mathrm{E}}[b(S(0), S(1), \ldots, S(\tau))],
$$

where tilde signifies that the parameter value of the geometric random variable $\tau$ is altered; see (5.5) below. Thus, our problem can be further reduced to the problem of evaluating

$$
\mathrm{E}[b(S(0), S(1), \ldots, S(\tau))]
$$

for arbitrary geometric random variables $\tau$ independent of the stock price process $\{S(t)\}$. For binomial and trinomial tree models, we have derived explicit expressions for (1.9) for $b$ being payoff functions of call options, put options, barrier options, and lookback options.

Geometric stopping of a random walk is the discrete counterpart of exponential stopping of a Lévy process. Sections 2 - 4 provide a self-contained exposition of results, which are the tools for the subsequent sections.

Let $M(\tau)$ denote the running maximum of $\{X(t)\}$ up to time $\tau$. We show that the random variables $M(\tau)$ and $[X(\tau)-M(\tau)]$ are independent. Hence, under the assumption that the random walk $\{X(t)\}$ is integer-valued, for integers $h$ and $j$ with $h \geq \max (0, j)$,

$$
\operatorname{Pr}\{X(\tau)=j, M(\tau)=h\}=\operatorname{Pr}\{M(\tau)=h\} \times \operatorname{Pr}\{X(\tau)-M(\tau)=j-h\} .
$$

To determine the two probabilities on the right-hand side (RHS) of (1.10), we find their probability generating functions by means of the identity

$$
\mathrm{E}\left[z^{X(\tau)}\right]=\mathrm{E}\left[z^{M(\tau)}\right] \times \mathrm{E}\left[z^{X(\tau)-M(\tau)}\right] .
$$

Details of this important step are given in Section 3. Similarly, we can find the joint probability

$$
\operatorname{Pr}\{X(\tau)=j, m(\tau)=h\}, \quad h \leq \min (0, j),
$$

where $m(\tau)$ denote the running minimum of $\{X(t)\}$ up to time $\tau$. These joint probabilities are useful for valuing barrier options; see Section 8

Many contracts have a finite expiry date. This problem may be handled by means of the time-honored actuarial method of Esscher transforms; see Section 10.

The Appendix gives two identities in the trinomial tree case for the joint probability of $X(T)=j$ and $M(T) \geq k$, where $T, j$ and $k$ are integers, with $T$ and $k$ nonnegative and $k \geq j$. In the binomial tree case, these two identities can be found in Föllmer and Schied (2011). 
In deriving our formulas, we do not make any assumption whether the expectation (1.2) is calculated with respect to a risk-neutral probability measure. With the payoff being a function of $K_{x}$, we are in an incomplete market situation where there is no unique choice of probability measure for valuation.

We should emphasize that results in this paper are not restricted to valuing death benefits. Instead of a time-until-death random variable, we can consider a time-untilcatastrophe random variable, and so on. A key assumption is that such a random variable is independent of the stock-price process $\{S(t)\}$.

There is a relatively large literature about equity-linked annuities. Excellent literature reviews can be found in Azimzadeh et al. (2014), Bacinello et al. (2011), and MacKay (2014).

\section{Geometric stopping of a random walk}

We consider a random walk with initial position $X(0)=0$ and independent and identically distributed (i.i.d.) increments $X_{1}, X_{2}, \ldots$ For $t=1,2, \ldots$, the position after $t$ steps is

$$
X(t)=X_{1}+\cdots+X_{t}
$$

Let $\tau$ be a geometric random variable (r.v.) independent of the random walk, with

$$
\operatorname{Pr}\{\tau=t\}=(1-\pi) \pi^{t}, \quad t=0,1,2, \ldots
$$

Its probability generating function (pgf) is

$$
P_{\tau}(z)=\sum_{t=0}^{\infty} \operatorname{Pr}\{\tau=t\} z^{t}=\frac{1-\pi}{1-\pi z} .
$$

We assume that each step of the random walk takes one time unit. We stop the random walk at time $\tau$ and are interested in $X(\tau)$. Its characteristic function is

$$
\mathrm{E}\left[e^{i y X(\tau)}\right]=\mathrm{E}\left[\mathrm{E}\left[e^{i y X(\tau)} \mid \tau\right]\right]=\mathrm{E}\left[\mathrm{E}\left[e^{i y X_{1}}\right]^{\tau}\right]=\frac{1-\pi}{1-\pi \mathrm{E}\left[e^{i y X_{1}}\right]},
$$

$y$ real. This determines the distribution of $X(\tau)$. Many actuaries would recognize (2.4) in the context of aggregate claims and interpret $X(\tau)$ as a compound geometric r.v. See (12.2.9) in Bowers et al. (1997) or (9.8) in Klugman et al. (2012).

We are also interested in the record highs and lows of the random walk. Let

$$
M(t)=\max \{0, X(1), \ldots, X(t)\}
$$

denote the running maximum up to step $t$ and, similarly, $m(t)$ the running minimum up to step $t$. We are interested in $M(\tau)$ and $m(\tau)$. We discuss two basic properties:

1. The random variables $M(\tau)$ and $X(\tau)-M(\tau)$ are independent. This is true because the conditional distribution of $[X(\tau)-M(\tau)]$, given $M(\tau)$, is the conditional 
distribution of $X(\tau)$, given $X(t) \leq 0$ for $t=1, \ldots, \tau$, and hence the same for all values of $M(\tau)$. To see this, consider the first time $t^{*}$ when $X\left(t^{*}\right)=M(\tau)$; thus $\tau \geq t^{*}$ and $\left[X(t)-X\left(t^{*}\right)\right] \leq 0$ for $t=t^{*}, \ldots, \tau$. Then observe that by the memoryless property of $\tau$, the conditional distribution of $\tau-t^{*}$ does not depend on $t^{*}$. Similarly, one shows that $m(\tau)$ and $X(\tau)-m(\tau)$ are independent.

2. The random variables $M(\tau)$ and $X(\tau)-m(\tau)$ have the same distribution. This is true because $M(t)$ and $X(t)-m(t)$ have the same distribution for each $t$. To see this, compare the following,

$$
\begin{aligned}
M(t) & =\max \left\{0, X_{1}, X_{1}+X_{2}, \ldots, X_{1}+X_{2}+\ldots+X_{t}\right\}, \\
X(t)-m(t) & =\max \left\{0, X_{t}, X_{t}+X_{t-1}, \ldots, X_{t}+X_{t-1}+\ldots+X_{1}\right\},
\end{aligned}
$$

and use the assumption that $X_{1}, X_{2}, \ldots$ are i.i.d. Similarly, one shows that $m(\tau)$ and $X(\tau)-M(\tau)$ have the same distribution.

From the decomposition

$$
X(\tau)=M(\tau)+[X(\tau)-M(\tau)]
$$

and the two properties above, it follows that, for each real $y$,

$$
\mathrm{E}\left[e^{i y X(\tau)}\right]=\mathrm{E}\left[e^{i y M(\tau)}\right] \mathrm{E}\left[e^{i y[X(\tau)-M(\tau)]}\right]=\mathrm{E}\left[e^{i y M(\tau)}\right] \mathrm{E}\left[e^{i y m(\tau)}\right],
$$

which can be viewed as a version of the celebrated Wiener-Hopf factorization.

The proofs for the two basic properties of the geometrically stopped random walk are accessible if not elementary. For the exponentially stopped Lévy process, two analogous properties hold. A proof of the first property is much more challenging from a technical point of view; see Bertoin (1996), Doney (2007), or Kyprianou (2006). Hence, the results for the geometrically stopped random walk are also helpful for a better understanding of those for the exponentially stopped Lévy process.

Remark 2.1: Formula (2.6) also follows from an identity due to Spitzer (1954, Theorem 6.1); see also Wendel (1958) and Kesten (1993). Let $x_{+}=\max \{0, x\}$ and $x_{-}=(-x)_{+}$. For $|w| \leq 1$ and $|z| \leq 1$, Spitzer's formula is

$$
\sum_{t=0}^{\infty} \pi^{t} \mathrm{E}\left[w^{M(t)} z^{M(t)-X(t)}\right]=\exp \left(\sum_{k=1}^{\infty} \frac{\pi^{k}}{k}\left\{\mathrm{E}\left[w^{X(k)_{+}}\right]+\mathrm{E}\left[z^{X(k)-}\right]-1\right\}\right),
$$

which can be rewritten as

$$
\begin{aligned}
& \sum_{t=0}^{\infty}(1-\pi) \pi^{t} \mathrm{E}\left[w^{M(t)} z^{M(t)-X(t)}\right] \\
& =\exp \left(\sum_{k=1}^{\infty} \frac{\pi^{k}}{k}\left\{\mathrm{E}\left[w^{X(k)+}\right]-1\right\}\right) \exp \left(\sum_{k=1}^{\infty} \frac{\pi^{k}}{k}\left\{\mathrm{E}\left[z^{X(k)}-\right]-1\right\}\right) .
\end{aligned}
$$

The left-hand side (LHS) of (2.8) is $\mathrm{E}\left[w^{M(\tau)} z^{M(\tau)-X(\tau)}\right]$. By considering $z=1$ or $w=1$, we see that the two factors on the RHS of $(2.8)$ are $\mathrm{E}\left[w^{M(\tau)}\right]$ and $\mathrm{E}\left[z^{M(\tau)-X(\tau)}\right]$. Hence, (2.6) is a consequence of Spitzer's identity. 


\section{$3 \quad$ Integer-valued increments}

We consider the special case where the increments are integer-valued and between $-m$ and $n(m \geq 1, n \geq 1)$. Let $p_{j}=\operatorname{Pr}\left\{X_{1}=j\right\}$; we assume $p_{-m} \neq 0$ and $p_{n} \neq 0$. The pgf of an increment is

$$
P_{X_{1}}(z)=\sum_{j=-m}^{n} p_{j} z^{j}
$$

Note that (2.4) can be written in terms of pgf's:

$$
P_{X(\tau)}(z)=\frac{1-\pi}{1-\pi P_{X_{1}}(z)}
$$

Similarly, (2.6) becomes

$$
P_{X(\tau)}(z)=P_{M(\tau)}(z) P_{m(\tau)}(z)
$$

Note that the RHS of (3.2) is a rational function. Let us write it as a ratio of two polynomials:

$$
P_{X(\tau)}(z)=\frac{(1-\pi) z^{m}}{g(z)}
$$

where

$$
g(z)=z^{m}\left[1-\pi P_{X_{1}}(z)\right]=z^{m}-\pi \sum_{j=-m}^{n} p_{j} z^{m+j}
$$

is a polynomial of degree $m+n$. Because for $|z|=1$,

$$
\left|z^{m}-g(z)\right|=\pi\left|\sum_{j=-m}^{n} p_{j} z^{m+j}\right| \leq \pi \sum_{j=-m}^{n} p_{j}\left|z^{m+j}\right|=\pi<\left|z^{m}\right|,
$$

it follows from Rouché's Theorem that $g(z)$ has the same number of zeros, counting multiplicity, inside the complex disk of radius 1 as the function $z^{m}$. Denote these $m$ zeros of $g(z)$ as $\alpha_{1}, \ldots, \alpha_{m}$. Denote the other zeros of $g(z)$, those with absolute value greater than 1 , as $\beta_{1}, \ldots, \beta_{n}$. Then, it follows from the condition $P_{X(\tau)}(1)=1$ that (3.4) can be written as

$$
P_{X(\tau)}(z)=z^{m}\left(\prod_{j=1}^{m} \frac{1-\alpha_{j}}{z-\alpha_{j}}\right)\left(\prod_{j=1}^{n} \frac{\beta_{j}-1}{\beta_{j}-z}\right) .
$$

The function $g(z)$ has exactly two positive zeros, one between 0 and 1 , the other greater than 1. To see this, consider the denominator of $(3.2)$ on the interval $(0, \infty)$. It is a continuous function of $z$, and its second derivative is negative. Thus it has at most two positive zeros. On the other hand, the denominator is positive for $z=0$ and tends to $-\infty$ at each of the two ends of the interval. 
Because $M(\tau) \geq 0, P_{M(\tau)}(z)$ exists for $|z|<1$. Similarly, $P_{m(\tau)}(z)$ exists for $|z|>1$. It follows from $(3.3),(3.7)$ and $P_{M(\tau)}(1)=P_{m(\tau)}(1)=1$ that

$$
P_{m(\tau)}(z)=\prod_{j=1}^{m} \frac{1-\alpha_{j}}{1-\alpha_{j} / z}
$$

and

$$
P_{M(\tau)}(z)=\prod_{j=1}^{n} \frac{1-1 / \beta_{j}}{1-z / \beta_{j}}
$$

By expanding (3.8), (3.9) and their product by partial fractions, we can identify the distributions of $M(\tau), m(\tau)$ and $X(\tau)$. We assume that the zeros of $g(z)$ are distinct. (If some of the zeros coincide, the following formulas would have to be amended accordingly.) Then, (3.9) can be rewritten as

$$
P_{M(\tau)}(z)=\sum_{k=1}^{n} \frac{\prod_{j=1}^{n}\left(1-1 / \beta_{j}\right)}{\prod_{j=1, j \neq k}^{n}\left(1-\beta_{k} / \beta_{j}\right)} \frac{1}{1-z / \beta_{k}} .
$$

It follows that

$$
\operatorname{Pr}\{M(\tau)=i\}=\sum_{k=1}^{n} \frac{\prod_{j=1}^{n}\left(1-1 / \beta_{j}\right)}{\prod_{j=1, j \neq k}^{n}\left(1-\beta_{k} / \beta_{j}\right)}\left(\frac{1}{\beta_{k}}\right)^{i}
$$

for $i=0,1,2, \ldots$ Similarly, we find that

$$
\operatorname{Pr}\{m(\tau)=i\}=\sum_{k=1}^{m} \frac{\prod_{j=1}^{m}\left(1-\alpha_{j}\right)}{\prod_{j=1, j \neq k}^{m}\left(1-\alpha_{j} / \alpha_{k}\right)}\left(\frac{1}{\alpha_{k}}\right)^{i}
$$

for $i=0,-1,-2, \ldots$ Also,

$$
\operatorname{Pr}\{X(\tau)=i\}=\sum_{k=1}^{m} \frac{\prod_{j=1}^{m}\left(1-\alpha_{j}\right)}{\prod_{j=1, j \neq k}^{m}\left(1-\alpha_{j} / \alpha_{k}\right)} \frac{\prod_{j=1}^{n}\left(1-1 / \beta_{j}\right)}{\prod_{j=1}^{n}\left(1-\alpha_{k} / \beta_{j}\right)}\left(\frac{1}{\alpha_{k}}\right)^{i},
$$

for $i=-1,-2, \ldots$, and

$$
\operatorname{Pr}\{X(\tau)=i\}=\sum_{k=1}^{n} \frac{\prod_{j=1}^{m}\left(1-\alpha_{j}\right)}{\prod_{j=1}^{m}\left(1-\alpha_{j} / \beta_{k}\right)} \frac{\prod_{j=1}^{n}\left(1-1 / \beta_{j}\right)}{\prod_{j=1, j \neq k}^{n}\left(1-\beta_{k} / \beta_{j}\right)}\left(\frac{1}{\beta_{k}}\right)^{i},
$$

for $i=0,1,2, \ldots$

To obtain the joint probabilities, we use properties 1 and 2 in Section 2. For $h=$ $0,1,2, \ldots$ and $j \leq h$ we have

$$
\begin{aligned}
\operatorname{Pr}\{X(\tau)=j, M(\tau)=h\} & =\operatorname{Pr}\{M(\tau)=h, X(\tau)-M(\tau)=j-h\} \\
& =\operatorname{Pr}\{M(\tau)=h\} \operatorname{Pr}\{X(\tau)-M(\tau)=j-h\} \\
& =\operatorname{Pr}\{M(\tau)=h\} \operatorname{Pr}\{m(\tau)=j-h\},
\end{aligned}
$$


which can be evaluated by means of (3.11) and (3.12). Similarly, for $k=0,-1,-2, \ldots$ and $j \geq k$ we have

$$
\operatorname{Pr}\{X(\tau)=j, m(\tau)=k\}=\operatorname{Pr}\{m(\tau)=k\} \operatorname{Pr}\{M(\tau)=j-k\}
$$

If the random walk is skip-free upwards, i.e., if $n=1$, there is another way to determine the distribution of $M(\tau)$. In this case, the event $M(\tau) \geq i$ is the same as the event that $X(t)=i$ for some $t \leq \tau$. Write $\beta$ for $\beta_{1}$. We stop the martingale $\left\{\beta^{X(t)} I_{(\tau \geq t)}\right\}$ at the first time $t^{*} \leq \tau$ when $X\left(t^{*}\right)=i$, or else at time $\tau+1$. Because $\beta>1$, we can apply the optional sampling theorem to obtain

$$
1=\beta^{i} \operatorname{Pr}\{M(\tau) \geq i\}
$$

Thus, for $i=0,1,2, \ldots$,

$$
\operatorname{Pr}\{M(\tau) \geq i\}=\left(\frac{1}{\beta}\right)^{i}
$$

and

$$
\operatorname{Pr}\{M(\tau)=i\}=\left(1-\frac{1}{\beta}\right)\left(\frac{1}{\beta}\right)^{i}
$$

which is indeed (3.11) with $n=1$. For the case where the random walk is skip-free downwards, i.e., $m=1$, we write $\alpha$ for $\alpha_{1}$, which is a number between 0 and 1 . We stop the martingale $\left\{\alpha^{X(t)} I_{(\tau \geq t)}\right\}$ at the first time $t^{*} \leq \tau$ when $X\left(t^{*}\right)=i$, or else at time $\tau+1$. The optional sampling theorem tells us that

$$
1=\alpha^{i} \operatorname{Pr}\{m(\tau) \leq i\}
$$

Thus, for $i=0,-1,-2, \ldots$,

$$
\operatorname{Pr}\{m(\tau) \leq i\}=\left(\frac{1}{\alpha}\right)^{i}
$$

and

$$
\operatorname{Pr}\{m(\tau)=i\}=(1-\alpha)\left(\frac{1}{\alpha}\right)^{i}
$$

which is (3.12) with $m=1$.

Let us look at the exit and overshoot problem for a finite interval. Let $k_{1}$ be a negative integer and $k_{2}$ a positive integer. We consider the first time $t^{*} \leq \tau$ when $X\left(t^{*}\right) \leq k_{1}$ or $X\left(t^{*}\right) \geq k_{2}$. Let $\Pi_{1 i}$ denote the probability that $X\left(t^{*}\right)=k_{1}-i(i=0,1, \ldots, m-1)$ and $\Pi_{2 i}$ the probability that $X\left(t^{*}\right)=k_{2}+i(i=0,1, \ldots, n-1)$. For $j=1,2, \ldots, m$, applying the optional sampling theorem to the martingale $\left\{\alpha_{j}^{X(t)} I_{(\tau \geq t)}\right\}$, we see that

$$
\sum_{i=0}^{m-1} \Pi_{1 i} \alpha_{j}^{k_{1}-i}+\sum_{i=0}^{n-1} \Pi_{2 i} \alpha_{j}^{k_{2}+i}=1 .
$$


Similarly, for $j=1,2, \ldots, n$,

$$
\sum_{i=0}^{m-1} \Pi_{1 i} \beta_{j}^{k_{1}-i}+\sum_{i=0}^{n-1} \Pi_{2 i} \beta_{j}^{k_{2}+i}=1 .
$$

These are $m+n$ linear equations for the $\Pi_{1 i}$ 's and $\Pi_{2 i}$ 's.

Remark 3.1: We note that $\operatorname{Pr}\{\tau \geq t\}=\pi^{t}$ can be interpreted as a $t$-period discount factor. Thus the $\Pi_{1 i}$ 's and $\Pi_{2 i}$ 's have an alternative interpretation as discounted probabilities of the overshoot at the time of the first exit from the interval. They can be used to compute the expected penalty or reward, if they are functions of the overshoot.

\section{The trinomial tree model}

In this section we consider the special case $m=n=1$. Then the zeros of $g(z)$, defined by (3.5), are the zeros of the quadratic polynomial

$$
\pi p_{1} z^{2}-\left(1-\pi p_{0}\right) z+\pi p_{-1} .
$$

We simplify the notation for the zeros by using the symbols $\alpha$ for $\alpha_{1}$ and $\beta$ for $\beta_{1}$. Note that

$$
0<\alpha<1<\beta
$$

Formula (3.2) can be written as

$$
P_{X(\tau)}(z)=\frac{1-\pi}{1-\pi\left(p_{1} z+p_{0}+p_{-1} z^{-1}\right)} .
$$

From (4.2) or from (3.13) and (3.14) we see that

$$
\begin{array}{ll}
\operatorname{Pr}\{X(\tau)=j\}=C \alpha^{-j}, & j=-1,-2, \ldots, \\
\operatorname{Pr}\{X(\tau)=j\}=C \beta^{-j}, & j=0,1,2, \ldots,
\end{array}
$$

with

$$
C=\frac{(1-\alpha)(\beta-1)}{\beta-\alpha}
$$

Thus $X(\tau)$ has a two-sided geometric distribution.

Formulas (3.17) - (3.20) are of course valid in this special case. From (3.15), (3.18) and (3.20) we obtain

$$
\operatorname{Pr}\{X(\tau)=j, M(\tau)=h\}=(1-\alpha)\left(1-\frac{1}{\beta}\right)\left(\frac{1}{\alpha}\right)^{j-h}\left(\frac{1}{\beta}\right)^{h},
$$

for $h=0,1,2, \ldots$ and $j \leq h$. Then, for $k=0,1,2, \ldots$

$$
\operatorname{Pr}\{X(\tau)=j, M(\tau) \geq k\}= \begin{cases}C\left(\frac{1}{\alpha}\right)^{j-k}\left(\frac{1}{\beta}\right)^{k}, & \text { if } j \leq k, \\ C\left(\frac{1}{\beta}\right)^{j}, & \text { if } j>k .\end{cases}
$$


Similarly, for $k=0,-1,-2, \ldots$ one finds that

$$
\operatorname{Pr}\{X(\tau)=j, m(\tau) \leq k\}= \begin{cases}C\left(\frac{1}{\alpha}\right)^{k}\left(\frac{1}{\beta}\right)^{j-k}, & \text { if } j \geq k, \\ C\left(\frac{1}{\alpha}\right)^{j}, & \text { if } j<k .\end{cases}
$$

From (4.4) with $j=0$ we see that $C=\operatorname{Pr}\{X(\tau)=0\}$. More generally, it follows from (4.7) and (3.17) that

$$
C=\operatorname{Pr}\{X(\tau)=k \mid M(\tau) \geq k\}
$$

$k=0,1,2, \ldots$ Similarly,

$$
C=\operatorname{Pr}\{X(\tau)=-k \mid m(\tau) \leq-k\}
$$

$k=0,1,2, \ldots$ If $(4.11)$ is written as

$$
\operatorname{Pr}\{X(\tau)=k \mid M(\tau) \geq k\}=\operatorname{Pr}\{X(\tau)=0\},
$$

it also holds for skip-free upward random walks. To see this, consider the first time when $X(t)=k$ and use the memoryless property of $\tau$. Similarly, if (4.12) is written as

$$
\operatorname{Pr}\{X(\tau)=-k \mid m(\tau) \leq-k\}=\operatorname{Pr}\{X(\tau)=0\}
$$

it also holds for skip-free downward random walks.

From (4.1), we have

$$
\alpha \beta=\frac{\pi p_{-1}}{\pi p_{1}}=\frac{p_{-1}}{p_{1}} .
$$

It follows from (4.7) and (4.4) that for $k \geq 0$ and $j \leq k$,

$$
\begin{aligned}
\operatorname{Pr}\{X(\tau)=j, M(\tau) \geq k\} & =C(\alpha \beta)^{k-j}\left(\frac{1}{\beta}\right)^{2 k-j} \\
& =\left(\frac{p_{-1}}{p_{1}}\right)^{k-j} \operatorname{Pr}\{X(\tau)=2 k-j\} .
\end{aligned}
$$

Similarly, it follows from (4.7) and (4.3) that for $k \geq 0$ and $j \leq k$,

$$
\operatorname{Pr}\{X(\tau)=j, M(\tau) \geq k\}=\left(\frac{p_{1}}{p_{-1}}\right)^{k} \operatorname{Pr}\{X(\tau)=-(2 k-j)\} .
$$

If $p_{0}=0$ (the binomial tree case) and if $\tau$ is replaced by a positive integer, the identities (4.16) and (4.17) can be found in Lemma 5.48 on page 298 of Föllmer and Schied (2011). In the next section, we shall see that the distribution of any nonnegative-integer-valued random variable can be approximated by linear combinations of geometric distributions. Hence, $\tau$ in (4.16) and (4.17) can be replaced by any nonnegative-integer-valued random variable independent of $\{X(t)\}$. A derivation of (4.16) and (4.17) by means of the reflection principle and the method of Esscher transforms can be found in the Appendix. 
Consider $0 \leq j \leq k$. Dividing (4.7) by (4.4) yields

$$
\operatorname{Pr}\{M(\tau) \geq k \mid X(\tau)=j\}=(\alpha / \beta)^{k-j} .
$$

Similarly, it follows from (4.9) and (4.3) that

$$
\operatorname{Pr}\{m(\tau) \leq-k \mid X(\tau)=-j\}=(\alpha / \beta)^{k-j} .
$$

Consequently, for $j=0,1,2, \ldots$ and $k \geq j$,

$$
\begin{aligned}
& \operatorname{Pr}\{M(\tau) \geq k \mid X(\tau)=j\}=\operatorname{Pr}\{M(\tau) \geq k-j \mid X(\tau)=0\}, \\
& \operatorname{Pr}\{m(\tau) \leq-k \mid X(\tau)=-j\}=\operatorname{Pr}\{m(\tau) \leq-(k-j) \mid X(\tau)=0\} .
\end{aligned}
$$

Identity (4.20) holds more generally for random walks that are skip-free upwards. To see this, consider the first time when $X(t)=j$ and use the memoryless property of $\tau$. Similarly, identity (4.21) holds more generally for random walks that are skip-free downwards.

Finally, let us consider the exit problem of Section 3 in the special case $m=n=1$. Let $\Pi_{1}$ be the probability that $X(t)$ reaches $k_{1}$ before or at time $\tau$, and before $X(t)=k_{2}$. Similarly, $\Pi_{2}$ is the probability that $X(t)$ reaches $k_{2}$ before or at time $\tau$, and before $X(t)=k_{1}$. According to (3.21) and (3.22)

$$
\begin{aligned}
\Pi_{1} \alpha^{k_{1}}+\Pi_{2} \alpha^{k_{2}} & =1 \\
\Pi_{1} \beta^{k_{1}}+\Pi_{2} \beta^{k_{2}} & =1
\end{aligned}
$$

It follows that

$$
\begin{aligned}
\Pi_{1} & =\frac{\beta^{k_{2}}-\alpha^{k_{2}}}{\alpha^{k_{1}} \beta^{k_{2}}-\alpha^{k_{2}} \beta^{k_{1}}}, \\
\Pi_{2} & =\frac{\alpha^{k_{1}}-\beta^{k_{1}}}{\alpha^{k_{1}} \beta^{k_{2}}-\alpha^{k_{2}} \beta^{k_{1}}} .
\end{aligned}
$$

Note that

$$
\lim _{k_{2} \rightarrow \infty} \Pi_{1}=\left(\frac{1}{\alpha}\right)^{k_{1}}
$$

which is consistent with (3.19), and that

$$
\lim _{k_{1} \rightarrow-\infty} \Pi_{2}=\left(\frac{1}{\beta}\right)^{k_{2}}
$$

which is consistent with (3.17).

\section{The valuation problem and its reduction}

We now turn to the evaluation of expected discounted values of the form (1.2). In a first step we choose an appropriate time unit. Let $K_{x}$ denote the curtate-future-lifetime r.v. 
(measured in number of time units) for a life-age- $x$. Upon death, a benefit payment $B$ is payable at time $K_{x}+1$. We assume a constant rate of interest and denote by $v$ the discount factor per unit time. The general goal is to calculate

$$
\mathrm{E}\left[v^{K_{x}+1} B\right]=\sum_{n=0}^{\infty} \operatorname{Pr}\left\{K_{x}=n\right\} v^{n+1} \mathrm{E}\left[B \mid K_{x}=n\right],
$$

the expectation of the discounted death benefit payment.

The random variable $B$ depends on a stock index or stock price, the time- $t$ value of which is denoted as $S(t), t=0,1, \ldots$ In general, $B$ can be a function of $S(0), S(1), \ldots$, $S\left(K_{x}\right)$,

$$
B=b\left(S(0), S(1), \ldots, S\left(K_{x}\right)\right) .
$$

We assume that $K_{x}$ and $\{S(t) ; t=0,1, \ldots\}$ are independent.

The distribution of $K_{x}$ can be approximated by distributions that are linear combinations of geometric distributions:

$$
\operatorname{Pr}\left\{K_{x}=n\right\} \approx \sum_{j} c_{j}\left(1-\pi_{j}\right) \pi_{j}^{n}, \quad n=0,1,2, \ldots,
$$

with $\sum_{j} c_{j}=1$. (Note that some of coefficients $c_{1}, c_{2}, \ldots$ can be negative.) To see this, consider the future-lifetime r.v. $T_{x}$. It is well-known (Dufresne 2007a, 2007b; Ko and Ng 2007) that its distribution can be approximated by distributions that are linear combinations of exponential distributions,

$$
\operatorname{Pr}\left\{T_{x}>t\right\} \approx \sum_{j} c_{j} e^{-\lambda_{j} t}, \quad t \geq 0
$$

with $\sum_{j} c_{j}=1$. Then,

$$
\operatorname{Pr}\left\{K_{x}=n\right\}=\operatorname{Pr}\left\{n \leq T_{x}<n+1\right\} \approx \sum_{j} c_{j}\left(1-e^{-\lambda_{j}}\right)\left(e^{-\lambda_{j}}\right)^{n} .
$$

With the substitution $\pi_{j}=e^{-\lambda_{j}}$, this yields (5.3).

As a consequence of (5.3), it suffices to consider the reduced problem where $K_{x}$ in (5.1) is a geometrically distributed r.v. $\tau$ which is independent of $\{S(t)\}$, that is, to calculate

$$
\mathrm{E}\left[v^{\tau+1} B\right]=\sum_{n=0}^{\infty}(1-\pi) \pi^{n} v^{n+1} \mathrm{E}[B \mid \tau=n] .
$$

We can simplify the problem by one more step:

$$
\begin{aligned}
\mathrm{E}\left[v^{\tau+1} B\right] & =\frac{v(1-\pi)}{1-v \pi} \sum_{n=0}^{\infty}(1-v \pi)(v \pi)^{n} \mathrm{E}[B \mid \tau=n] \\
& =\frac{v(1-\pi)}{1-v \pi} \tilde{\mathrm{E}}[B] \\
& =\mathrm{E}\left[v^{\tau+1}\right] \tilde{\mathrm{E}}[B]
\end{aligned}
$$


Formally, there is no discounting in the expectation $\tilde{\mathrm{E}}[B]$. But note that the expectation is taken with respect to the geometric distribution with parameter $v \pi$ instead of $\pi$. Some actuaries would recognize this change of distribution as an Esscher transform. We shall refer (5.5) as a factorization formula. See also Remark 10.4.

Remark 5.1: There is a more general factorization formula for the LHS of (5.1). We note that

$$
\mathrm{E}\left[v^{K_{x}+1} B\right]=\mathrm{E}\left[v^{K_{x}+1}\right] \times \sum_{n=0}^{\infty} \widetilde{\operatorname{Pr}}\left\{K_{x}=n\right\} \mathrm{E}\left[B \mid K_{x}=n\right]
$$

with the changed probabilities

$$
\widetilde{\operatorname{Pr}}\left\{K_{x}=n\right\}=\frac{v^{n} \operatorname{Pr}\left\{K_{x}=n\right\}}{\mathrm{E}\left[v^{K_{x}}\right]}, \quad n=0,1,2, \ldots
$$

Hence,

$$
\mathrm{E}\left[v^{K_{x}+1} B\right]=\mathrm{E}\left[v^{K_{x}+1}\right] \times \tilde{\mathrm{E}}[B]
$$

where the last expectation is taken with respect to the changed probabilities (5.7).

\section{$6 \quad$ The stock price model}

To complete the description of the problem, we specify the model for $\{S(t) ; t=0,1,2, \ldots\}$. In the rest of this paper, we assume that for some $a>1$,

$$
S(t)=S(0) a^{X(t)}, \quad t=0,1,2, \ldots,
$$

where $\{X(t) ; t=0,1,2, \ldots\}$ is a random walk of Section 4 . That is, we assume a trinomial tree model for stock price movements. (Many authors write $a$ as $u$ and $1 / a$ as $d$.) Thanks to the factorization formula (5.5), we can use the results of Section 4 , if $\pi$ is replaced by $v \pi$. The quadratic polynomial (4.1) is replaced by

$$
v \pi p_{1} z^{2}-\left(1-v \pi p_{0}\right) z+v \pi p_{-1}
$$

In this section, we abuse notation to also denote the zeros of (6.2) as $\alpha$ and $\beta, 0<\alpha<$ $1<\beta$. Note that $\alpha$ and $\beta$ are the two values of $z$ for which the stochastic process,

$$
\left\{v^{t} z^{X(t)} I_{(\tau \geq t)}, \quad t=0,1,2 \ldots\right\},
$$

is a martingale.

As a first example, we consider $B=S(\tau)$. By (5.5) and (4.2) with $z=a$, we have

$$
\begin{aligned}
\mathrm{E}\left[v^{\tau+1} S(\tau)\right] & =\frac{v(1-\pi)}{1-v \pi} \tilde{\mathrm{E}}[S(\tau)] \\
& =\frac{v(1-\pi)}{1-v \pi} S(0) \tilde{\mathrm{E}}\left[a^{X(\tau)}\right] \\
& =S(0) \frac{v(1-\pi)}{1-v \pi\left(p_{1} a+p_{0}+p_{-1} a^{-1}\right)} .
\end{aligned}
$$


In order that this is finite, we assume here and in the following that

$$
v \pi\left(p_{1} a+p_{0}+p_{-1} a^{-1}\right)<1
$$

or equivalently, that $\beta>a$; recall that $\beta$ is the larger of the two zeros of (6.2) and that $a>1$. Note that by (3.7), the expectation $\tilde{\mathrm{E}}\left[a^{X(\tau)}\right]$ is also

$$
a \frac{(1-\alpha)(\beta-1)}{(a-\alpha)(\beta-a)}
$$

If $v$ is the one-period risk-free discount factor, $p_{1}, p_{0}$ and $p_{-1}$ are risk-neutral probabilities, and the stock pays no dividends, then (6.4) reduces to $\pi<1$, which is always true.

The Brownian motion can be approximated by (and obtained as a limit from) trinomial random walks. Let $\{W(t), t \geq 0\}$ be a Brownian motion with drift $\mu$ and diffusion coefficient $\sigma$. A first idea is to construct a crude approximation for $W(t)$ by using $\kappa X(\lfloor t\rfloor)$, where $\kappa=\ln a>0$ is the amplitude of the jumps. Here, $\lfloor x\rfloor$ denotes the greatest integer less than or equal to $x$. Matching the first two moments of $W(1)$ and $\kappa X(1)$ yields two conditions:

$$
-\kappa p_{-1}+\kappa p_{1}=\mu, \quad \kappa^{2} p_{-1}+\kappa^{2} p_{1}=\sigma^{2}+\mu^{2} .
$$

The second condition means

$$
\kappa=\sqrt{\frac{\sigma^{2}+\mu^{2}}{1-p_{0}}} .
$$

The first condition is satisfied by setting

$$
p_{-1}=\frac{1-p_{0}}{2}-\frac{\mu}{2 \kappa}, \quad p_{1}=\frac{1-p_{0}}{2}+\frac{\mu}{2 \kappa} .
$$

If we impose the additional condition of matching third moments,

$$
-\kappa^{3} p_{-1}+\kappa^{3} p_{1}=3 \mu \sigma^{2}+\mu^{3}
$$

then the solution is unique, with

$$
p_{0}=\frac{2 \sigma^{2}}{3 \sigma^{2}+\mu^{2}}
$$

A better approximation can be expected, if $W(t)$ is approximated by

$$
\kappa X(\lfloor n t\rfloor)
$$

with $n$ sufficiently large. (Of course, $\kappa$ depends on $n$.) To use (6.5) and (6.6), we must replace $\mu$ by $\mu / n$ and $\sigma^{2}$ by $\sigma^{2} / n$ on their RHS. As $n$ is large, it is reasonable to set

$$
\kappa=\frac{\sigma}{\sqrt{n\left(1-p_{0}\right)}}
$$


Hence (6.6) becomes

$$
p_{-1}=\frac{1-p_{0}}{2}-\frac{\mu \sqrt{1-p_{0}}}{2 \sigma \sqrt{n}}, \quad p_{1}=\frac{1-p_{0}}{2}+\frac{\mu \sqrt{1-p_{0}}}{2 \sigma \sqrt{n}}
$$

We would mention two prominent special cases. The first case is the binomial tree of Cox et al. (1979, p. 249). Here, $p_{0}=0$, and (6.9) and (6.10) become

$$
\kappa=\sigma \sqrt{\frac{1}{n}}, \quad p_{-1}=\frac{1}{2}-\frac{\mu}{2 \sigma} \sqrt{\frac{1}{n}}, \quad p_{1}=\frac{1}{2}+\frac{\mu}{2 \sigma} \sqrt{\frac{1}{n}} .
$$

The second case is the trinomial tree of Hull (2012, p. 444), where the value of $p_{0}$ is determined by considering (6.7), with $\mu$ replaced by $\mu / n, \sigma^{2}$ replaced by $\sigma^{2} / n$, and $n$ sufficiently large. Thus, $p_{0}=2 / 3$, and

$$
\kappa=\sigma \sqrt{\frac{3}{n}}, \quad p_{-1}=\frac{1}{6}-\frac{\mu}{6 \sigma} \sqrt{\frac{3}{n}}, \quad p_{1}=\frac{1}{6}+\frac{\mu}{6 \sigma} \sqrt{\frac{3}{n}} .
$$

Results in Gerber et al. (2012) can be obtained as limits from results in this paper by considering

$$
S(t)=S(0) e^{\kappa X(\lfloor n t\rfloor)}, \quad t \geq 0,
$$

with $\kappa$ defined by (6.9) and the probabilities of down and up moves given by (6.10). This continuous-time stock price model has up to $n$ jumps per unit time. Formulas in this paper are usually given for the case of one period per unit time. Hence, we would replace $v$ by $v^{1 / n}=e^{-\delta / n}$ and $\pi$ by $\pi^{1 / n}=e^{-\lambda / n}$, where $\delta$ denotes the force of interest and $\lambda$ is the parameter of the exponential stopping time in the continuous model in Gerber et al. (2012).

Remark 6.1: Consider the geometric two-Poisson stock price model,

$$
S(t)=S(0) e^{\kappa\left[N_{2}(t)-N_{1}(t)\right]}, \quad t \geq 0,
$$

where $N_{1}(t)$ and $N_{2}(t)$ are independent Poisson processes with parameters $\lambda_{1}$ and $\lambda_{2}$, respectively. The two-Poisson process can be obtained as a limit of trinomial random walks by setting

$$
p_{-1}=\lambda_{1} / n, \quad p_{1}=\lambda_{2} / n, \quad p_{0}=1-\left(\lambda_{1}+\lambda_{2}\right) / n .
$$

Thus several results of this paper can be used to obtain results for the geometric twoPoisson stock price model. Of course this model is not nearly as popular as the classical geometric Brownian motion model. But note that the latter can be obtained as a limit from the former. For example, set

$$
\lambda_{1}=\frac{\sigma^{2}-\kappa \mu}{2 \kappa^{2}}, \quad \lambda_{2}=\frac{\sigma^{2}+\kappa \mu}{2 \kappa^{2}}, \quad \kappa \rightarrow 0
$$




\section{$7 \quad B$ a function of $S\left(K_{x}\right)$}

Consider a death benefit payment $B$ that is a function of the stock index at the beginning of the time interval in which $(x)$ dies,

$$
B=b\left(S\left(K_{x}\right)\right)
$$

for some function $b(s)$. Because of the approximation formula (5.3) and the factorization formula (5.5), it is sufficient to calculate

$$
\begin{aligned}
\mathrm{E}[B] & =\mathrm{E}[b(S(\tau))] \\
& =\sum_{j=-\infty}^{\infty} b\left(S(0) a^{j}\right) \operatorname{Pr}\{X(\tau)=j\} \\
& =\sum_{j=-\infty}^{-1} b\left(S(0) a^{j}\right) C \alpha^{-j}+\sum_{j=0}^{\infty} b\left(S(0) a^{j}\right) C \beta^{-j}
\end{aligned}
$$

The last equality is due to (4.3) and (4.4). We introduce the function

$$
\mathcal{E}_{b}(s)=\mathrm{E}\left[b\left(s a^{X(\tau)}\right)\right], \quad s>0 .
$$

Then the expectation $\mathrm{E}[B]$ can be written as $\mathcal{E}_{b}(S(0))$. We shall now consider some special cases where the RHS of (7.2) can be simplified.

For $b(s)=I_{(s \leq K)}$, the payoff function of a cash-or-nothing put option, it follows from (7.2) that

$$
\mathcal{E}_{b}(S(0))= \begin{cases}C \frac{(1 / \alpha)^{\ell}}{1-\alpha}, & \text { if } S(0)>K \\ 1-C \frac{(1 / \beta)^{\ell+1}}{1-1 / \beta}, & \text { if } S(0) \leq K\end{cases}
$$

where

$$
\ell=\left\lfloor\log _{a} \frac{K}{S(0)}\right\rfloor
$$

is the integer part of $\log _{a} \frac{K}{S(0)}$.

For $b(s)=s I_{(s \leq K)}$, the payoff function of an asset-or-nothing put option, it follows from (7.2) that

$$
\mathcal{E}_{b}(S(0))= \begin{cases}S(0) C \frac{(a / \alpha)^{\ell}}{1-\alpha / a}, & \text { if } S(0)>K, \\ \mathrm{E}[S(\tau)]-S(0) C \frac{(a / \beta)^{\ell+1}}{1-a / \beta}, & \text { if } S(0) \leq K\end{cases}
$$

By (2.4),

$$
\mathrm{E}[S(\tau)]=S(0) \frac{1-\pi}{1-\pi\left(p_{1} a+p_{0}+p_{-1} a^{-1}\right)}
$$

in the trinomial tree model, and

$$
\mathrm{E}[S(\tau)]=S(0) \frac{1-\pi}{1-\pi\left(p a+q a^{-1}\right)}
$$


in the binomial tree model.

For the put option with exercise price $K, b(s)=(K-s)_{+}$. Instead of determining $\mathcal{E}_{b}(S(0))$ by $(7.2)$, we can use the identity

$$
(K-s)_{+}=K I_{(s \leq K)}-s I_{(s \leq K)}
$$

Hence,

$$
\mathcal{E}_{b}(S(0))= \begin{cases}K C \frac{(1 / \alpha)^{\ell}}{1-\alpha}-S(0) C \frac{(a / \alpha)^{\ell}}{1-\alpha / a}, & \text { if } S(0)>K, \\ K-K C \frac{(1 / \beta)^{\ell+1}}{1-1 / \beta}-\mathrm{E}[S(\tau)]+S(0) C \frac{(a / \beta)^{\ell+1}}{1-a / \beta}, & \text { if } S(0) \leq K,\end{cases}
$$

the difference of $K$ times (7.4) and (7.6).

For $b(s)=I_{(s>K)}$, the payoff function of a cash-or-nothing call option, it follows from (7.2) that

$$
\mathcal{E}_{b}(S(0))= \begin{cases}C \frac{(1 / \beta)^{\ell+1}}{1-1 / \beta}, & \text { if } S(0) \leq K \\ 1-C \frac{(1 / \alpha)^{\ell}}{1-\alpha}, & \text { if } S(0)>K\end{cases}
$$

For $b(s)=s I_{(s>K)}$, the payoff function of an asset-or-nothing call option, it follows from (7.2) that

$$
\mathcal{E}_{b}(S(0))= \begin{cases}S(0) C \frac{(a / \beta)^{\ell+1}}{1-a / \beta}, & \text { if } S(0) \leq K \\ \mathrm{E}[S(\tau)]-S(0) C \frac{(a / \alpha)^{\ell}}{1-\alpha / a}, & \text { if } S(0)>K\end{cases}
$$

For the call option with exercise price $K, b(s)=(s-K)_{+}$. Instead of determining $\mathcal{E}_{b}(S(0))$ by $(7.2)$, we can use the identity

$$
(s-K)_{+}=s I_{(s>K)}-K I_{(s>K)} .
$$

Hence,

$$
\mathcal{E}_{b}(S(0))= \begin{cases}S(0) C \frac{(a / \beta)^{\ell+1}}{1-a / \beta}-K C \frac{(1 / \beta)^{\ell+1}}{1-1 / \beta}, & \text { if } S(0) \leq K, \\ \mathrm{E}[S(\tau)]-S(0) C \frac{(a / \alpha)^{\ell}}{1-\alpha / a}-K+K C \frac{(1 / \alpha)^{\ell}}{1-\alpha}, & \text { if } S(0)>K\end{cases}
$$

the difference of (7.12) and $K$ times (7.11).

Remark 7.1: As a check, note that the difference between the RHS of (7.14) and that of (7.10) equals $\mathrm{E}[S(\tau)]-K$, in accordance to put-call parity.

\section{Barrier options}

For the knock-in options, $B=b\left(S\left(K_{x}\right)\right)$ if the stock price reaches one or two predetermined barriers before or at time $\tau$; otherwise $B=0$. 
First we consider a double barrier option with barriers $L<U$ and initial stock price $S(0)$ with $L<S(0)<U$. We may assume that

$$
k_{1}=\log _{a}\left(\frac{L}{S(0)}\right), \quad k_{2}=\log _{a}\left(\frac{U}{S(0)}\right)
$$

are integers. The probability that the stock price first reaches the lower end $L$ of the barrier interval, and that this takes place before or at time $\tau$, is $\Pi_{1}$ given by (4.24). At that time, because of the memoryless property of the geometric distribution, the conditional expectation of $B$ is $\mathcal{E}_{b}(L)$; where $\mathcal{E}_{b}(s)$ is defined by (7.3). Similarly, the probability that the stock price first reaches the upper end $U$ of the barrier interval, and that this happens before or at time $\tau$, is $\Pi_{2}$ given by (4.25). Then the conditional expectation of $B$ is $\mathcal{E}_{b}(U)$. Hence, by conditioning we find that

$$
\mathrm{E}[B]=\Pi_{1} \mathcal{E}_{b}(L)+\Pi_{2} \mathcal{E}_{b}(U) .
$$

We remember that for the call and put options, closed-form expressions are available for $\mathcal{E}_{b}(s)$; see (7.10) and (7.14).

Now we turn to the single barrier options. The down-and-in option comes into existence if the stock price reaches the level $L$ before or at time $\tau$, the probability of which is given by (3.19) with $k=k_{1}$. Thus

$$
\mathrm{E}[B]=\alpha^{-k_{1}} \mathcal{E}_{b}(L)
$$

In contrast, the up-and-in option comes into existence if the stock price reaches the level $U$ before or at time $\tau$, the probability of which is given by (3.17) with $k=k_{2}$. Thus

$$
\mathrm{E}[B]=\beta^{-k_{2}} \mathcal{E}_{b}(U) .
$$

We note that (8.3) and (8.4) can be obtained as limiting formulas from (8.2), if $U \rightarrow \infty$ or $L \rightarrow 0$, respectively. Also, (8.3) holds more generally for random walks $\{X(t)\}$ that are skip-free downwards; as in Section 3, $\alpha$ stands for $\alpha_{1}$. Similarly, with $\beta=\beta_{1},(8.4)$ holds more generally for random walks $\{X(t)\}$ that are skip-free upwards.

A knock-out option exists at time 0 and goes out of existence when the stock price reaches the predetermined barrier(s). The payoff $B$ of a knock-out option is the payoff of the corresponding ordinary option reduced by the payoff of the corresponding knock-in option. From this and (8.2) - (8.4) it follows that

$$
\mathrm{E}[B]=\mathcal{E}_{b}(S(0))-\Pi_{1} \mathcal{E}_{b}(L)-\Pi_{2} \mathcal{E}_{b}(U)
$$

for the knock-out double barrier option,

$$
\mathrm{E}[B]=\mathcal{E}_{b}(S(0))-\Pi_{1} \mathcal{E}_{b}(L)
$$

for the down-and-out option, and

$$
\mathrm{E}[B]=\mathcal{E}_{b}(S(0))-\Pi_{2} \mathcal{E}_{b}(U)
$$

for the up-and-out option. 
Remark 8.1: Let $\theta_{1}=\log _{a}(\alpha)$ and $\theta_{2}=\log _{a}(\beta)$. It then follows from (8.1) that

$$
\alpha^{-k_{1}}=\left[\frac{L}{S(0)}\right]^{-\theta_{1}}, \quad \beta^{-k_{2}}=\left[\frac{U}{S(0)}\right]^{-\theta_{2}},
$$

with which the dependence on the current stock and barriers in (8.3) and (8.4) can be seen more clearly. It turns out that $\theta_{1}$ and $\theta_{2}$ are the two values of $\theta$ for which the stochastic process,

$$
\left\{[S(t)]^{\theta} I_{(\tau \geq t)}, \quad t=0,1,2, \ldots\right\},
$$

is a martingale. Stopping these two martingales at the first time when $S(t)$ reaches $L$ or $U$, and using the optional sampling theorem, we obtain

$$
\begin{aligned}
& S(0)^{\theta_{1}}=L^{\theta_{1}} \Pi_{1}+U^{\theta_{1}} \Pi_{2}, \\
& S(0)^{\theta_{2}}=L^{\theta_{2}} \Pi_{1}+U^{\theta_{2}} \Pi_{2} .
\end{aligned}
$$

By Cramer's rule,

$$
\begin{aligned}
\Pi_{1} & =\frac{\operatorname{det}\left[\begin{array}{ll}
S(0)^{\theta_{1}} & U^{\theta_{1}} \\
S(0)^{\theta_{2}} & U^{\theta_{2}}
\end{array}\right]}{\operatorname{det}\left[\begin{array}{ll}
L^{\theta_{1}} & U^{\theta_{1}} \\
L^{\theta_{2}} & U^{\theta_{2}}
\end{array}\right]}, \\
\Pi_{2} & =\frac{\operatorname{det}\left[\begin{array}{ll}
L^{\theta_{1}} & S(0)^{\theta_{1}} \\
L^{\theta_{2}} & S(0)^{\theta_{2}}
\end{array}\right]}{\operatorname{det}\left[\begin{array}{ll}
L^{\theta_{1}} & U^{\theta_{1}} \\
L^{\theta_{2}} & U^{\theta_{2}}
\end{array}\right]},
\end{aligned}
$$

which are more meaningful than (4.24) and (4.25) in the context of the stock price reaching a barrier. From (8.10) and (8.11), we can immediately see that if $S(0)=L$, we have $\Pi_{1}=1$ and $\Pi_{2}=0$, and if $S(0)=U$, we have $\Pi_{1}=0$ and $\Pi_{2}=1$.

Remark 8.2: For the single barrier options the expected payoff can also be obtained from (4.7) - (4.10). For example, for the up-and-in option, it is

$$
\begin{aligned}
& \sum_{j=-\infty}^{\infty} b\left(S(0) a^{j}\right) \operatorname{Pr}\left\{X(\tau)=j, M(\tau) \geq k_{2}\right\} \\
= & \sum_{j=-\infty}^{k_{2}} b\left(S(0) a^{j}\right) C \alpha^{-j}\left(\frac{\alpha}{\beta}\right)^{k_{2}}+\sum_{j=k_{2}+1}^{\infty} b\left(S(0) a^{j}\right) C \beta^{-j} .
\end{aligned}
$$

To see that this is the same as (8.4), rewrite (8.4) as

$$
\mathrm{E}[B]=\beta^{-k_{2}} \mathcal{E}_{b}\left(S(0) a^{k_{2}}\right)
$$

and apply (7.3) and (7.2).

Remark 8.3: In general, out-of-the-money put and call options can be viewed as single barrier options, with the barrier being the strike price. For example, the outof-the money call option in Section 7 is an up-and-in barrier option with $U=K$ and 
$b(s)=(s-K)_{+}$. Let us check that the two expressions for $\mathrm{E}[B]$ are indeed equivalent. We start with the upper expression on the RHS of (7.14). It follows from (7.5) and (8.1) that $\ell=k_{2}$. Because $S(0) a^{\ell}=K$, the expression can be written as

$$
\beta^{-\ell}\left[K C \frac{a / \beta}{1-a / \beta}-K C \frac{1 / \beta}{1-1 / \beta}\right] .
$$

With $S(0)=K$ in (7.14) and in (7.5), we see that the expression inside the bracket of $(8.13)$ is $\mathcal{E}_{b}(K)$. Thus, for $S(0) \leq K$, we have

$$
\mathcal{E}_{b}(S(0))=\beta^{-\ell} \mathcal{E}_{b}(K)
$$

which is the RHS of (8.4) with $U=K$.

Remark 8.4: In addition to knock-in and knock-out options, there is a third kind of barrier options known as rebate options. A rebate option makes a fixed payment, if the stock price reaches a barrier before the option expires. The payment is made at the moment when the barrier is reached. (If the payment is made at the expiry date, the option is called a deferred rebate option.) With $\alpha$ and $\beta$ being the zeros of (6.2), $\alpha^{-k_{1}}$ is the expected present value of the contingent payment of 1 from a down rebate option if level $L$ is reached before or at time $\tau$, and $\beta^{-k_{2}}$ is the expected present value of the contingent payment of 1 from an up rebate option if level $U$ is reached before or at time $\tau$. (Also note (8.8).) Similarly, $\Pi_{1}$ and $\Pi_{2}$ are also expected present values of rebate payoffs. Rebates are usually associated with knock-out options, as compensations when the options are knocked-out. Here, we can use the concept of rebate options to value knock-in options because $\tau$ has the memoryless property.

Remark 8.5: Barrier options can be used to incorporate lapses and surrenders. As we discussed in the Introduction, a put option becomes less valuable as the stock price rises. Because the policy may lapse, we might consider the up-and-out put option payoff (1.6), which can now be rewritten as

$$
I_{\left(S(0) a^{M\left(K_{x}\right)}<H\right)} \times\left[G-S\left(K_{x}\right)\right]_{+} .
$$

Of course, the value of (8.14) is lower than that of (1.4). The higher is the stock price, the more likely a policy will lapse. A refinement of (8.14) is a weighted average of up-and-out put option payoffs,

$$
\sum_{j} \pi_{j} I_{\left(S(0) a^{M\left(K_{x}\right)}<H_{j}\right)} \times\left[G-S\left(K_{x}\right)\right]_{+},
$$

where $S(0)<H_{1}<H_{2}<H_{3}<\ldots$ and $\pi_{1}, \pi_{2}, \pi_{3}, \ldots$ are positive numbers that sum to one. Two recent publications that extensively discuss lapses and surrenders are Campbell et al. (2014) and MacKay (2014).

Remark 8.6: In practice, the price of the underlying asset of a barrier option or lookback option is not monitored continuously, but periodically. This is certainly an argument in favor of a discrete-time model. 


\section{Lookback options}

Many equity-indexed annuities contain lookback, high water mark or low water mark options (Streiff and DiBiase 1999; Tiong 2000). In this section we provide closed-form formulas for various lookback options.

\subsection{Fixed strike lookback call option}

The benefit payment or payoff $B$ is

$$
\left[\max \left(H, \max _{0 \leq t \leq \tau} S(t)\right)-K\right]_{+}=\left[\max \left(H, S(0) a^{M(\tau)}\right)-K\right]_{+} .
$$

Here, $H$ is a positive constant with $H \geq S(0)$; it can be interpreted as the maximum level of the stock's historical $(t \leq 0)$ prices. To value this payoff, we need to distinguish whether the strike price $K$ is higher or lower than the historical maximum price $H$, that is, we need to distinguish whether the option is out-of-the money or in-the-money.

For the out-of-the-money case $(K>H)$, the payoff $(9.1)$ simplifies as

$$
\left[S(0) a^{M(\tau)}-K\right]_{+}
$$

By (3.18),

$$
\begin{aligned}
\mathrm{E}\left[\left[S(0) a^{M(\tau)}-K\right]_{+}\right] & =\sum_{k=\ell+1}\left[S(0) a^{k}-K\right](\beta-1) \beta^{-k-1} \\
& =S(0) \frac{\beta-1}{\beta} \frac{(a / \beta)^{\ell+1}}{1-a / \beta}-K(1 / \beta)^{\ell+1} .
\end{aligned}
$$

For the in-the-money case $(K<H)$, the payoff $(9.1)$ is

$$
\max \left(H, S(0) a^{M(\tau)}\right)-K .
$$

By rewriting (9.4) as

$$
H-K+\left[S(0) a^{M(\tau)}-H\right]_{+}
$$

and using (9.3) with $K$ replaced by $H$, we find that the expectation of (9.4) is

$$
H-K+S(0) \frac{\beta-1}{\beta} \frac{(a / \beta)^{\ell+1}}{1-a / \beta}-H(1 / \beta)^{\ell+1}
$$

\subsection{Fixed strike lookback put option}

The benefit payment or payoff $B$ is

$$
\left[K-\min \left(H, \min _{0 \leq t \leq \tau} S(t)\right)\right]_{+}=\left[K-\min \left(H, S(0) a^{m(\tau)}\right)\right]_{+} .
$$


Here, $H$ is a positive constant, with $H \leq S(0)$; it can be interpreted as the minimum level of the stock's historical $(t<0)$ prices. To value this payoff, we need to distinguish whether the strike price $K$ is lower or higher than the historical minimum price $H$, that is, we need to distinguish whether the option is out-of-the money or in-the-money.

For the out-of-the-money case $(K<H)$, the payoff $(9.7)$ simplifies as

$$
\left[K-S(0) a^{m(\tau)}\right]_{+}
$$

By $(3.20)$,

$$
\mathrm{E}\left[\left[K-S(0) a^{m(\tau)}\right]_{+}\right]=K(1 / \alpha)^{\ell}-S(0)(1-\alpha) \frac{(a / \alpha)^{\ell}}{1-\alpha / a}
$$

For the in-the-money case $(K>H)$, the payoff $(9.7)$ is

$$
K-\min \left(H, S(0) a^{m(\tau)}\right)=K-H+\left[H-S(0) a^{m(\tau)}\right]_{+},
$$

whose expectation is

$$
K-H+H(1 / \alpha)^{\ell}-S(0)(1-\alpha) \frac{(a / \alpha)^{\ell}}{1-\alpha / a} .
$$

\subsection{Floating strike lookback call option}

The benefit payment or payoff $B$ is

$$
S(\tau)-\min \left(H, \min _{0 \leq t \leq \tau} S(t)\right)
$$

where $0<H \leq S(0)$. By comparing (9.12) with the LHS of (9.10), we see that the expectation of (9.12) is (9.11) with $K$ replaced by $\mathrm{E}[S(\tau)]$, namely,

$$
\mathrm{E}[S(\tau)]-H+H(1 / \alpha)^{\ell}-S(0)(1-\alpha) \frac{(a / \alpha)^{\ell}}{1-\alpha / a} .
$$

\subsection{Floating strike lookback put option}

The benefit payment or payoff $B$ is

$$
\max \left(H, \max _{0 \leq t \leq \tau} S(t)\right)-S(\tau)
$$

where $H \geq S(0)$. By comparing (9.14) with (9.4), we see that its expectation is (9.6) but with $K$ replaced by $\mathrm{E}[S(\tau)]$. The result is

$$
H-\mathrm{E}[S(\tau)]+S(0) \frac{\beta-1}{\beta} \frac{(a / \beta)^{\ell+1}}{1-a / \beta}-H(1 / \beta)^{\ell+1} .
$$




\subsection{Fractional floating strike lookback call option}

The benefit payment or payoff $B$ is

$$
\begin{aligned}
{\left[S(\tau)-\gamma \min _{0 \leq t \leq \tau} S(t)\right]_{+} } & =S(0)\left[a^{X(\tau)}-\gamma a^{m(\tau)}\right]_{+} \\
& =S(0) a^{m(\tau)}\left[a^{(X(\tau)-m(\tau))}-\gamma\right]_{+}
\end{aligned}
$$

where $\gamma \geq 1$. By noting that $m(\tau)$ and $[X(\tau)-m(\tau)]$ are independent and by applying (9.3), the expectation of (9.16) is

$$
S(0) \frac{1-\alpha}{1-\alpha / a}\left[\frac{\beta-1}{\beta} \frac{(a / \beta)^{\left\lfloor\log _{a} \gamma\right\rfloor+1}}{1-a / \beta}-\gamma(1 / \beta)^{\left\lfloor\log _{a} \gamma\right\rfloor+1}\right] .
$$

\subsection{Fractional floating strike lookback put option}

The benefit payment or payoff $B$ is

$$
\left[\gamma \max _{0 \leq t \leq \tau} S(t)-S(\tau)\right]_{+}=S(0)\left[\gamma a^{M(\tau)}-a^{X(\tau)}\right]_{+}=S(0) a^{M(\tau)}\left[\gamma-a^{X(\tau)-M(\tau)}\right]_{+},
$$

where $0<\gamma \leq 1$. By noting that $M(\tau)$ and $[X(\tau)-M(\tau)]$ are independent and by applying (9.9), the expectation of (9.18) is

$$
S(0) \frac{\beta-1}{\beta-a}\left[\gamma(1 / \alpha)^{\left\lfloor\log _{a} \gamma\right\rfloor}-(1-\alpha) \frac{(a / \alpha)^{\left\lfloor\log _{a} \gamma\right\rfloor}}{1-\alpha / a}\right] .
$$

\subsection{High-low option}

The high-low option is also called the length-of-range option. Its benefit payment or payoff $B$ is

$$
\max \left(\bar{H}, \max _{0 \leq t \leq \tau} S(t)\right)-\min \left(\underline{H}, \min _{0 \leq t \leq \tau} S(t)\right)
$$

where $0<\underline{H} \leq S(0) \leq \bar{H}$. The parameters $\underline{H}$ and $\bar{H}$ can be interpreted as the past stock-price minimum and maximum, respectively. We note that the payoff (9.20) is the sum of (9.14) with $H=\bar{H}$ and (9.12) with $H=\underline{H}$. Hence it follows from (9.15) and (9.13) that the expectation of (9.20) is

$$
\begin{aligned}
& \bar{H}+S(0) \frac{\beta-1}{\beta} \frac{(a / \beta)^{\ell+1}}{1-a / \beta}-\bar{H}(1 / \beta)^{\ell+1} \\
& -\underline{H}+\underline{H}(1 / \alpha)^{\ell}-S(0)(1-\alpha) \frac{(a / \alpha)^{\ell}}{1-\alpha / a} .
\end{aligned}
$$




\section{Expiry at time $T$}

Suppose that $b\left(S\left(K_{x}\right)\right)$ is payable at time $K_{x}+1$, provided that $K_{x}<T$, where $T$ is a fixed positive integer. This is the situation of a $T$-year term insurance contract. Because of (5.3) and (5.5), it is sufficient to determine

$$
\mathrm{E}\left[b(S(\tau)) I_{(\tau<T)}\right]=\mathrm{E}[b(S(\tau))]-\mathrm{E}\left[b(S(\tau)) I_{(\tau \geq T)}\right] .
$$

The last term in (10.1) is

$$
\begin{aligned}
\mathrm{E}\left[b(S(\tau)) I_{(\tau \geq T)}\right] & =\operatorname{Pr}\{\tau \geq T\} \mathrm{E}[b(S(\tau)) \mid \tau \geq T] \\
& =\pi^{T} \mathrm{E}\left[b\left(S(T) a^{X(\tau)-X(T)}\right) \mid \tau \geq T\right] \\
& =\pi^{T} \mathrm{E}\left[\mathrm{E}\left[b\left(S(T) a^{X(\tau)-X(T)}\right) \mid \tau \geq T, S(T)\right]\right] .
\end{aligned}
$$

Because $\tau$ has the memoryless property and is independent of the random walk $\{X(t)\}$, we see that

$$
\begin{aligned}
& \mathrm{E}\left[b\left(S(T) a^{X(\tau)-X(T)}\right) \mid \tau \geq T, S(T)\right] \\
& =\mathrm{E}\left[b\left(S(T) a^{X\left(\tau^{*}\right)}\right) \mid S(T)\right]=\mathcal{E}_{b}(S(T)),
\end{aligned}
$$

where $\tau^{*}$ has the same distribution as $\tau$ and is also independent of $\{X(t)\}$. Hence

$$
\mathrm{E}\left[b(S(\tau)) I_{(\tau \geq T)}\right]=\pi^{T} \mathrm{E}\left[\mathcal{E}_{b}(S(T))\right] .
$$

So the remaining task is to calculate $\mathrm{E}\left[\mathcal{E}_{b}(S(T))\right]$.

For each of the $b(s)$ functions considered in Section 7, a formula for $\mathrm{E}\left[\mathcal{E}_{b}(S(T))\right]$ is given below. These results will be explained and justified after this paragraph. For $b(s)=I_{(s \leq K)}$,

$$
\begin{aligned}
\mathrm{E}\left[\mathcal{E}_{b}(S(T))\right]= & C \frac{(1 / \alpha)^{\ell}}{1-\alpha} \mathrm{E}\left[\alpha^{X(T)}\right] \operatorname{Pr}\{S(T)>K ; \alpha\} \\
& +\operatorname{Pr}\{S(T) \leq K\}-C \frac{(1 / \beta)^{\ell+1}}{1-1 / \beta} \mathrm{E}\left[\beta^{X(T)}\right] \operatorname{Pr}\{S(T) \leq K ; \beta\},
\end{aligned}
$$

where $\ell$ is defined by $(7.5)$. For $b(s)=s I_{(s \leq K)}$,

$$
\begin{aligned}
\mathrm{E}\left[\mathcal{E}_{b}(S(T))\right]= & C \frac{(a / \alpha)^{\ell}}{1-\alpha / a} S(0) \mathrm{E}\left[\alpha^{X(T)}\right] \operatorname{Pr}\{S(T)>K ; \alpha\} \\
& +\mathrm{E}[S(\tau)] \mathrm{E}\left[a^{X(T)}\right] \operatorname{Pr}\{S(T) \leq K ; a\} \\
& -C \frac{(a / \beta)^{\ell+1}}{1-a / \beta} S(0) \mathrm{E}\left[\beta^{X(T)}\right] \operatorname{Pr}\{S(T) \leq K ; \beta\} .
\end{aligned}
$$

For $b(s)=(K-s)_{+}$,

$$
\begin{aligned}
\mathrm{E}\left[\mathcal{E}_{b}(S(T))\right]= & C\left[K \frac{(1 / \alpha)^{\ell}}{1-\alpha}-S(0) \frac{(a / \alpha)^{\ell}}{1-\alpha / a}\right] \mathrm{E}\left[\alpha^{X(T)}\right] \operatorname{Pr}\{S(T)>K ; \alpha\} \\
& +C\left[S(0) \frac{(a / \beta)^{\ell+1}}{1-a / \beta}-K \frac{(1 / \beta)^{\ell+1}}{1-1 / \beta}\right] \mathrm{E}\left[\beta^{X(T)}\right] \operatorname{Pr}\{S(T) \leq K ; \beta\} \\
& +K \operatorname{Pr}\{S(T) \leq K\}-\mathrm{E}[S(\tau)] \mathrm{E}\left[a^{X(T)}\right] \operatorname{Pr}\{S(T) \leq K ; a\} .
\end{aligned}
$$


For $b(s)=I_{(s>K)}$,

$$
\begin{aligned}
\mathrm{E}\left[\mathcal{E}_{b}(S(T))\right]= & C \frac{(1 / \beta)^{\ell+1}}{1-1 / \beta} \mathrm{E}\left[\beta^{X(T)}\right] \operatorname{Pr}\{S(T) \leq K ; \beta\} \\
& +\operatorname{Pr}\{S(T)>K\}-C \frac{(1 / \alpha)^{\ell}}{1-\alpha} \mathrm{E}\left[\alpha^{X(T)}\right] \operatorname{Pr}\{S(T)>K ; \alpha\} .
\end{aligned}
$$

For $b(s)=s I_{(s>K)}$,

$$
\begin{aligned}
\mathrm{E}\left[\mathcal{E}_{b}(S(T))\right]= & C \frac{(a / \beta)^{\ell+1}}{1-a / \beta} S(0) \mathrm{E}\left[\beta^{X(T)}\right] \operatorname{Pr}\{S(T) \leq K ; \beta\} \\
& +\mathrm{E}[S(\tau)] \mathrm{E}\left[a^{X(T)}\right] \operatorname{Pr}\{S(T)>K ; a\} \\
& -C \frac{(a / \alpha)^{\ell}}{1-\alpha / a} S(0) \mathrm{E}\left[\alpha^{X(T)}\right] \operatorname{Pr}\{S(T)>K ; \alpha\} .
\end{aligned}
$$

For $b(s)=(s-K)_{+}$,

$$
\begin{aligned}
\mathrm{E}\left[\mathcal{E}_{b}(S(T))\right]= & C\left[S(0) \frac{(a / \beta)^{\ell+1}}{1-a / \beta}-K \frac{(1 / \beta)^{\ell+1}}{1-1 / \beta}\right] \mathrm{E}\left[\beta^{X(T)}\right] \operatorname{Pr}\{S(T) \leq K ; \beta\} \\
& +C\left[K \frac{(1 / \alpha)^{\ell}}{1-\alpha}-S(0) \frac{(a / \alpha)^{\ell}}{1-\alpha / a}\right] \mathrm{E}\left[\alpha^{X(T)}\right] \operatorname{Pr}\{S(T)>K ; \alpha\} \\
& -K \operatorname{Pr}\{S(T)>K\}+\mathrm{E}[S(\tau)] \mathrm{E}\left[a^{X(T)}\right] \operatorname{Pr}\{S(T)>K ; a\} .
\end{aligned}
$$

We shall now explain these results. The probabilities in (10.4) - (10.9) refer to Esscher transformed probabilities of $X(T)$. For $h>0$, they are defined by

$$
\operatorname{Pr}\{X(T)=j ; h\}=h^{j} \operatorname{Pr}\{X(T)=j\} / \mathrm{E}\left[h^{X(T)}\right] .
$$

An immediate consequence is the factorization formula for Esscher transforms:

$$
\mathrm{E}\left[h^{X(T)} f(X(T))\right]=\mathrm{E}\left[h^{X(T)}\right] \mathrm{E}[f(X(T)) ; h] .
$$

See Gerber and Shiu (1994). Note that

$$
\mathrm{E}\left[h^{X(T)}\right]=\left(\mathrm{E}\left[h^{X_{1}}\right]\right)^{T},
$$

which is $\left(p_{1} h+p_{0}+p_{-1} h^{-1}\right)^{T}$ in the trinomial tree model and $\left(p h+q h^{-1}\right)^{T}$ in the binomial tree model.

Because $\mathrm{E}\left[\mathcal{E}_{b}(S(0))\right]=\mathcal{E}_{b}(S(0))$, it is no surprise that with $T=0$, formulas (10.4) to (10.9) are (7.4), (7.6), (7.10), (7.11), (7.12), and (7.14), respectively. For the converse, i.e., for deriving the formula for $\mathrm{E}\left[\mathcal{E}_{b}(S(T))\right]$ from the corresponding $\mathcal{E}_{b}(S(0))$ formula, we evaluate

$$
\mathrm{E}\left[\mathcal{E}_{b}(S(T))\right]=\mathrm{E}\left[\mathcal{E}_{b}(S(T)) I_{(S(T) \leq K)}\right]+\mathrm{E}\left[\mathcal{E}_{b}(S(T)) I_{(S(T)>K)}\right] .
$$

Because $S(0)$ is to be replaced by $S(T)=S(0) a^{X(T)}, \ell$ is replaced by

$$
\left\lfloor\log _{a} \frac{K}{S(T)}\right\rfloor=\left\lfloor\log _{a} \frac{K}{S(0)}+\log _{a} \frac{S(0)}{S(T)}\right\rfloor=\left\lfloor\log _{a} \frac{K}{S(0)}\right\rfloor-X(T)=\ell-X(T) .
$$


As an example for the verification, we now show how the first term on the RHS of (10.4) follows from the first line of (7.4). Here, $b(s)=I_{(s \leq K)}$. We have indeed

$$
\begin{aligned}
& \mathrm{E}\left[\mathcal{E}_{b}(S(T)) I_{(S(T)>K)}\right] \\
& =C \frac{(1 / \alpha)^{\ell}}{1-\alpha} \mathrm{E}\left[\alpha^{X(T)} I_{(S(T)>K)}\right] \\
& =C \frac{(1 / \alpha)^{\ell}}{1-\alpha} \mathrm{E}\left[\alpha^{X(T)}\right] \operatorname{Pr}\{S(T)>K ; \alpha\} .
\end{aligned}
$$

Remark 10.1: As a check, note that the difference between the RHS of (10.9) and that of (10.6) equals

$$
\mathrm{E}[S(\tau)] \mathrm{E}\left[a^{X(T)}\right]-K=\mathrm{E}[S(T)] \mathrm{E}\left[a^{X(\tau)}\right]-K
$$

in accordance to put-call parity.

Remark 10.2: Under the Esscher transform, the increments $\left\{X_{j}\right\}$ remain i.i.d, with modified probabilities

$$
\operatorname{Pr}\left\{X_{1}=j ; h\right\}=\frac{h^{j}}{\mathrm{E}\left[h^{X_{1}}\right]} \operatorname{Pr}\left\{X_{1}=j\right\}=\frac{h^{j}}{\mathrm{E}\left[h^{X_{1}}\right]} p_{j} .
$$

In the binomial tree model, the modified probabilities are

$$
\operatorname{Pr}\left\{X_{1}=1 ; h\right\}=\frac{p h}{p h+q h^{-1}}, \quad \operatorname{Pr}\left\{X_{1}=-1 ; h\right\}=\frac{q h^{-1}}{p h+q h^{-1}} .
$$

Remark 10.3: The factorization formula (10.11) remains valid if $f(X(T))$ is replaced by a function that depends on $X(1), X(2), \ldots, X(T)$. For example, the function can depend on $M(T)$ or $m(T)$ or both. Let us write such a function or functional as $f_{T}(X)$. Then,

$$
\mathrm{E}\left[h^{X(T)} f_{T}(X)\right]=\mathrm{E}\left[h^{X(T)}\right] \mathrm{E}\left[f_{T}(X) ; h\right] .
$$

Remark 10.4: It follows from the law of iterated expectations and formula (2.2) that

$$
\mathrm{E}\left[h^{X(\tau)}\right]=\mathrm{E}\left[\mathrm{E}\left[h^{X_{1}}\right]^{\tau}\right]=\frac{1-\pi}{1-\pi \mathrm{E}\left[h^{X_{1}}\right]} .
$$

Thus, the factorization formula (10.17) can be generalized as

$$
\mathrm{E}\left[h^{X(\tau)} f_{\tau}(X)\right]=\mathrm{E}\left[h^{X(\tau)}\right] \mathrm{E}\left[f_{\tau}(X) ; h\right],
$$

where, however,

$$
\operatorname{Pr}\{\tau=t ; h\}=\left(1-\pi_{h}\right)\left(\pi_{h}\right)^{t}, \quad t=0,1,2, \ldots,
$$

with

$$
\pi_{h}=\pi \mathrm{E}\left[h^{X_{1}}\right] .
$$


Here, $\alpha$ and $\beta$ are replaced by $\alpha / h$ and $\beta / h$, the solutions of

$$
1=\pi_{h} \times \mathrm{E}\left[z^{X_{1}} ; h\right]=\pi_{h} \times \frac{\mathrm{E}\left[(h z)^{X_{1}}\right]}{\mathrm{E}\left[h^{X_{1}}\right]}=\pi \times \mathrm{E}\left[(h z)^{X_{1}}\right] .
$$

If $\pi_{h}<1$, we still have $\alpha / h<1<\beta / h$. From condition (6.4), we have $\pi_{a}<1$.

Remark 10.5: As an application of (10.19), let us show that we can derive the assetor-nothing call option formula (7.12) from the cash-or-nothing call option formula (7.11). From (10.19) we have

$$
\mathrm{E}\left[S(\tau) I_{(S(\tau)>K)}\right]=\mathrm{E}[S(\tau)] \operatorname{Pr}\{S(\tau)>K ; a\} .
$$

Comparing (7.12) with (7.11) and noting (4.5), we see that it remains to show

$$
\mathrm{E}[S(\tau)] \frac{\left(1-\alpha a^{-1}\right)\left(\beta a^{-1}-1\right)}{\beta a^{-1}-\alpha a^{-1}}=S(0) \frac{(1-\alpha)(\beta-1)}{\beta-\alpha},
$$

or

$$
\mathrm{E}\left[a^{X(\tau)}\right]=\frac{(1-\alpha)(\beta-1)}{(a-\alpha)(\beta-a)} a
$$

which corresponds to (3.7) with $m=n=1$ and $z=a$.

\section{Conclusion}

The goal of this paper is the valuation of the expected present value of death benefits that are a function of the price or price history of a stock or stock fund. The logarithm of the stock price process is modeled as a discrete random walk. The policyholder's future lifetime is assumed to be independent of the stock price process. Because the distribution of the curtate-future-lifetime random variable can be approximated by linear combinations of geometric distributions, it suffices to consider curtate-future-lifetimes with a geometric distribution. That is, the evaluation problem reduces to the special case where the force of mortality is constant. A key tool is a discrete-time analog of the continuous-time Wiener-Hopf factorization approach used in Lévy processes exit problems. The paper is self-contained, as the theoretical machinery needed in the discrete model is much simpler than that in the continuous model. For binomial and trinomial tree models, we have derived closed-form formulas for the expected present value of the following options that are exercised at the end of the period of death: call options, put options, barrier options, and lookback options. Option Greeks and higher moments can also be readily determined.

The evaluation of equity-linked insurance and annuity products normally requires the use of computer systems. Closed-form formulas are especially useful for testing whether the computer system has been programmed correctly. 


\section{Acknowledgments}

We are grateful to Daniel Dufresne, X. Sheldon Lin, Zhenhao Zhou, and the anonymous referees for their valuable insights. We acknowledge with thanks the support from the Principal Financial Group Foundation, Research Grants Council of the Hong Kong Special Administrative Region (project No. HKU 705313P), and Society of Actuaries' Centers of Actuarial Excellence Research Grants.

\section{References}

Azimzadeh, P., Forsyth, P. A., Vetzal, K. R., 2014. Hedging costs for variable annuities under regime-switching. In: Mamon, R.S., Elliott, R.J., (Eds.). Hidden Markov Models in Finance. New York: Springer, 133-166.

Bacinello, A.R., Millossovich, P., Olivieri, A., Pitacco, E., 2011. Variable annuities: a unifying valuation approach. Insurance: Mathematics and Economics 49: 285-297.

Bertoin, J. 1996. Lévy Processes. Cambridge University Press.

Bowers, N. L., Gerber, H.U., Hickman, J.C., Jones, D.A., Nesbitt, C.J., 1997. Actuarial Mathematics. 2nd edition, Schaumburg, Ill.: Society of Actuaries.

Campbell, J., Chan, M., Li, K., Lombardi, L., Lombardi, L., Purushotham, M., Rao, A., 2014. Modeling of Policyholder Behavior for Life Insurance and Annuity Products: A Survey and Literature Review. Society of Actuaries.

Cox, J.C., Ross, S.A., Rubinstein, M., 1979. Option pricing: a simplified approach. J. Financial Economics 7: 229-263.

Doney, R.A., 2007. Fluctuation Theory of Lévy Processes. Lecture Notes in Mathematics, Vol. 1897. Springer.

Dufresne, D., 2007a. Fitting combinations of exponentials to probability distributions. Applied Stochastic Models in Business and Industry 23: 23-48.

Dufresne, D., 2007b. Stochastic life annuities. North American Actuarial Journal 11(1): 136-157.

Föllmer, H., Schied, A., 2011. Stochastic Finance: An Introduction in Discrete Time. 3rd edition. De Gruyter.

Gerber, H.U., Shiu, E.S.W., 1994. Option pricing by Esscher transforms. Transactions of the Society of Actuaries 46: 99-140. Discussions 141-191.

Gerber, H.U., Shiu, E.S.W., Yang, H., 2012. Valuing equity-linked death benefits and other contingent options: a discounted density approach. Insurance: Mathematics and Economics 51(1): 73-92. 
Gerber, H.U., Shiu, E.S.W., Yang, H., 2013. Valuing equity-linked death benefits in jump diffusion models. Insurance: Mathematics and Economics 53(3): 615-623.

International Actuarial Association, 2010. Stochastic Modeling: Theory and Reality from an Actuarial Perspective. Ottawa, Canada: International Actuarial Association.

Hull, J., 2012. Options, Futures and other Derivatives, 8th edition, Prentice Hall.

Kesten, H., 1993. Frank Spitzer's work on random walk and Brownian motion. Annals of Probability, 21(2): 593-607.

Klugman, S.A., Panjer, H.H., Willmot, G.E., 2012. Loss Models: From Data to Decisions. 4th edition, Wiley.

Ko, B., Ng, A.C.Y., 2007. Discussion on "Stochastic life annuities" by D. Dufresne. North American Actuarial Journal 11(3): 170-171.

Kyprianou, A.E., 2006. Introductory Lectures on Fluctuations of Lévy Processes with Applications. Springer.

MacKay, A., 2014. Fee Structure and Surrender Incentives in Variable Annuities. Ph.D. Thesis, University of Waterloo.

Milevsky, M.A., Posner, S.E., 2001. The Titanic option: valuation of the guaranteed minimum death benefit in variable annuities and mutual funds. The Journal of Risk and Insurance 68 (1): 93-128.

Spitzer, F., 1956. A combinatorial lemma and its application to probability theory. Transactions of the American Mathematical Society 82(2): 323 - 339.

Streiff, T.F., DiBiase, C.A., 1999. Equity Indexed Annuities. Chicago: Dearborn.

Tiong, S., 2000. Valuing equity-indexed annuities. North American Actuarial Journal 4(4): 149-163. Discussions 4(4): 163-170; 5(3) (2001): 128-136.

Ulm, E.R., 2006. The effect of the real option to transfer on the value of guaranteed minimum death benefits. The Journal of Risk and Insurance, 73(1): 43-69.

Ulm, E.R., 2008. Analytic solution for return of premium and rollup guaranteed minimum death benefit options under some simple mortality laws. ASTIN Bulletin, 38(2): 543-563.

Wendel, J.G., 1958. Spitzer's formula: a short proof. Proceedings of the American Mathematical Society 9(6): 905-908. 


\section{Appendix: Joint distribution identities in the trino- mial tree model}

In this Appendix, we derive two identities, which are equivalent to (4.16) and (4.17), by means of the reflection principle and the method of Esscher transforms. As in Section 4, we consider the trinomial tree model. Let $T, k$ and $j$ be integers, with $T$ and $k$ being nonnegative and $k \geq j$. The two identities are:

$$
\begin{aligned}
& \operatorname{Pr}\{X(T)=j, M(T) \geq k\}=\left(p_{-1} / p_{1}\right)^{k-j} \operatorname{Pr}\{X(T)=2 k-j\}, \\
& \operatorname{Pr}\{X(T)=j, M(T) \geq k\}=\left(p_{1} / p_{-1}\right)^{k} \operatorname{Pr}\{X(T)=-(2 k-j)\} .
\end{aligned}
$$

First, we consider the symmetric trinomial tree case, i.e., $p_{1}=p_{-1}$. Then, by the reflection principle,

$$
\begin{aligned}
\operatorname{Pr}\{X(T)=j, M(T) \geq k\} & =\operatorname{Pr}\{X(T)=k+(k-j)\} \\
& =\operatorname{Pr}\{X(T)=2 k-j\} .
\end{aligned}
$$

Because $p_{1}=p_{-1}$, we also have the identity

$$
\operatorname{Pr}\{X(T)=j, M(T) \geq k\}=\operatorname{Pr}\{X(T)=-(2 k-j)\} .
$$

Next, we consider the general trinomial tree case (i.e., $p_{1}$ is not necessarily the same as $\left.p_{-1}\right)$. We use the method of Esscher transforms. Formula (10.15) means

$$
\operatorname{Pr}\left\{X_{1}=j ; h\right\} \propto h^{j} p_{j}
$$

Hence, under the Esscher transform indexed by the parameter

$$
h^{*}=\sqrt{p_{-1} / p_{1}}
$$

the r.v. $X_{1}$ is symmetric, i.e.,

$$
\operatorname{Pr}\left\{X_{1}=1 ; h^{*}\right\}=\operatorname{Pr}\left\{X_{1}=-1 ; h^{*}\right\} .
$$

Let $\left\{X_{j}^{*}\right\}$ denote a sequence of i.i.d. r.v.'s with

$$
\operatorname{Pr}\left\{X_{j}^{*}=n\right\}=\operatorname{Pr}\left\{X_{j}=n ; h^{*}\right\} .
$$

Note that

$$
\operatorname{Pr}\left\{X_{j}^{*}=n ; 1 / h^{*}\right\}=\operatorname{Pr}\left\{X_{j}=n\right\} .
$$

Define $X^{*}(t)=\sum_{j=1}^{t} X_{j}^{*}$ and let $M^{*}(t)$ denote its running maximum up to time $t$. Then identities (A.3) and (A.4) are valid with $X(t)$ replaced by $X^{*}(t)$ and $M(t)$ replaced by $M^{*}(t)$. Now,

$$
\begin{aligned}
\operatorname{Pr}\{X(T)=j, M(T) \geq k\} & =\mathrm{E}\left[I_{(X(T)=j, M(T) \geq k)}\right] \\
& =\mathrm{E}\left[I_{\left(X^{*}(T)=j, M^{*}(T) \geq k\right)} ; 1 / h^{*}\right] \\
& =\frac{\mathrm{E}\left[\left(1 / h^{*}\right)^{X^{*}(T)} I_{\left(X^{*}(T)=j, M^{*}(T) \geq k\right)}\right]}{\mathrm{E}\left[\left(1 / h^{*}\right)^{X^{*}(T)}\right]} .
\end{aligned}
$$


The numerator in (A.9) is

$$
\begin{aligned}
& \mathrm{E}\left[\left(1 / h^{*}\right)^{X^{*}(T)} I_{\left(X^{*}(T)=j, M^{*}(T) \geq k\right)}\right] \\
& =\left(1 / h^{*}\right)^{j} \mathrm{E}\left[I_{\left(X^{*}(T)=j, M^{*}(T) \geq k\right)}\right] \\
& =\left(1 / h^{*}\right)^{j} \mathrm{E}\left[I_{\left(X^{*}(T)=2 k-j\right)}\right] \\
& =\left(1 / h^{*}\right)^{j-(2 k-j)} \mathrm{E}\left[\left(1 / h^{*}\right)^{X^{*}(T)} I_{\left(X^{*}(T)=2 k-j\right)}\right] .
\end{aligned}
$$

It follows from (A.9), (A.10) and (A.6) that

$$
\begin{aligned}
\operatorname{Pr}\{X(T)=j, M(T) \geq k\} & =\left(1 / h^{*}\right)^{-2(k-j)} \mathrm{E}\left[I_{\left(X^{*}(T)=2 k-j\right)} ; 1 / h^{*}\right] \\
& =\left(p_{-1} / p_{1}\right)^{k-j} \mathrm{E}\left[I_{(X(T)=2 k-j)}\right]
\end{aligned}
$$

which is (A.1).

To obtain (A.10), we used (A.3) with $X(t)$ replaced by $X^{*}(t)$ and $M(t)$ replaced by $M^{*}(t)$. Instead of (A.3), we can use (A.4). Then, instead of (A.1) we have (A.2). Because the factor $\left(p_{1} / p_{-1}\right)^{k}$ does not involve $j$, identity (A.2) is equivalent to

$$
\operatorname{Pr}\{X(T) \leq j \& M(T) \geq k\}=\left(p_{1} / p_{-1}\right)^{k} \operatorname{Pr}\{X(T) \leq j-2 k\}, \quad k \geq \max (j, 0) .
$$

Remark: Recall the continuous-time stochastic processes $\{\kappa X(\lfloor n t\rfloor), t \geq 0\}$ introduced in (6.8), with $\kappa, p_{-1}$ and $p_{1}$ defined by (6.9) and (6.10). In the limit $n \rightarrow \infty$, it is the Brownian motion, $\{W(t), t \geq 0\}$, with drift $\mu$ and diffusion coefficient $\sigma$. We claim that

$$
\operatorname{Pr}\left\{W(t) \leq x \& M_{W}(t) \geq y\right\}=e^{R y} \operatorname{Pr}\{W(t) \leq x-2 y\}, \quad y \geq \max (x, 0),
$$

where $M_{W}(t)=\max \{W(s), 0 \leq s \leq t\}$, and $R=2 \mu / \sigma^{2}$. We use $R$ because it is the usual symbol for the adjustment coefficient in actuarial risk theory. To obtain (A.13), we use (A.12) with $j=\lfloor x / \kappa\rfloor, k=\lfloor y / \kappa\rfloor$, and $T=\lfloor n t\rfloor$. Thus

$$
\begin{aligned}
& \operatorname{Pr}\{X(\lfloor n t\rfloor) \leq\lfloor x / \kappa\rfloor, M(\lfloor n t\rfloor) \geq\lfloor y / \kappa\rfloor\} \\
& =\left[p_{1} / p_{-1}\right]^{\lfloor y / \kappa\rfloor} \operatorname{Pr}\{X(\lfloor n t\rfloor) \leq\lfloor x / \kappa\rfloor-2\lfloor y / \kappa\rfloor\} .
\end{aligned}
$$

Using (6.9) and (6.10), we have

$$
\lim _{n \rightarrow \infty}\left[p_{1} / p_{-1}\right]^{\lfloor y / \kappa\rfloor}=\lim _{n \rightarrow \infty}\left[1+\frac{2 \mu}{\sigma \sqrt{n} \sqrt{1-p_{0}}}+o(1 / \sqrt{n})\right]^{\left\lfloor y \sqrt{n\left(1-p_{0}\right)} / \sigma\right\rfloor}=e^{2 \mu y / \sigma^{2}} .
$$

Hence (A.13) is obtained as a limit of (A.14). 